\title{
BRCA1/MAD2L1 deficiency disrupts the spindle assembly checkpoint to confer vinorelbine resistance in mesothelioma
}

Sara Busacca ${ }^{1}$, Laura O' Regan², Anita Singh², Annabel J. Sharkey ${ }^{1}$, Alan G. Dawson, Joanna Dzialo ${ }^{1}$, Aimee Parsons ${ }^{1}$, Neelam Kumar ${ }^{3}$, Laurel M. Schunselaar ${ }^{4}$, Naomi Guppy ${ }^{5}$, Apostolos Nakas ${ }^{6}$, Michael Sheaff ${ }^{7}$, Aaron S. Mansfield ${ }^{8}$, Sam M Janes $^{3}$, Paul Baas ${ }^{4}$, Andrew M. Fry ${ }^{2}$ and Dean A. Fennell ${ }^{1^{*}}$

\section{Affiliation of Authors}

${ }^{1}$ Leicester Cancer Research Centre, University of Leicester, Leicester, UK

${ }^{2}$ Department of Molecular and Cell Biology, University of Leicester, Leicester, UK

${ }^{3}$ University College London, UCL Respiratory, London, UK

${ }^{4}$ Department of Thoracic Oncology, Netherlands Cancer Institute, Amsterdam, The Netherlands

${ }^{5}$ University College London Advanced Diagnostics, London, UK

6 Department of Thoracic Surgery, Glenfield Hospital, University Hospitals of Leicester, Leicester, UK

${ }^{7}$ Department of Histopathology, Barts Health NHS Trust, London, UK

${ }^{8}$ Department of Oncology, Division of Medical Oncology, Mayo Clinic, Rochester, Minnesota, USA.

Running title: BRCA1/MAD2L1 expression predicts response to vinorelbine 
${ }^{*}$ Corresponding author: Dean A. Fennell, University of Leicester \& University Hospitals of Leicester NHS Trust, Roberk Kilpatrick Building, Leicester Royal Infirmary, LE2 7LX, Leicester, UK. Email: df132@leicester.ac.uk

\section{Conflict of interest statement:}

D. A. Fennell reports grants from Astex Therapeutics, personal fees from Aldeyra, grants from Boehringer Ingelheim, non-financial support from Clovis, non-financial support from Eli Lilly, from BMS, personal fees from Inventiva, personal fees from Paredox, personal fees and non-financial support from Roche, grants from MSD, grants from Bayer, during the conduct of the study.

A. S. Mansfield has received research support from Novartis, and Verily; remuneration to his institution for participation in advisory boards or other activities from AstraZeneca, Bristol-Myers Squibb, F. Hoffmann-La Roche (including Genentech), and Abbvie; travel support from Abbvie and F. Hoffmann-La Roche; and is a non-remunerated director of the Mesothelioma Applied Research Foundation.

P Baas reports grants and other from BMS, grants and other from MSD, other from Aldeyra, other from Pfizer, other from AZ, outside the submitted work.

All other authors declare no potential conflicts of interest. 


\section{Abstract}

Mesothelioma is a universally lethal cancer lacking effective therapy. The spindle poison vinorelbine exhibits clinical activity in relapsed setting, and in preclinical models requires BRCA1 to initiate apoptosis. However, the mechanisms underlying this regulation and the clinical implications have not been explored.

Here we show that BRCA1 silencing abrogated vinorelbine-induced cell cycle arrest, recruitment of BUBR1 to kinetochores, and apoptosis. BRCA1 silencing led to codepletion of MAD2L1 at the mRNA and protein levels consistent with its status as a transcriptional target of BRCA1. Silencing of MAD2L1 phenocopied BRCA1, and was sufficient to confer resistance to vinorelbine. This was recapitulated in cell lines selected for resistance to vinorelbine, which acquired loss of both BRCA1 and MAD2L1 expression. Following ex-vivo vinorelbine in 20 primary tumour explants, apoptotic response rate was 59\% in BRCA1/MAD2L1 positive explants compared with $0 \%$ in BRCA1/MAD2L1 negative explants. In 48 patients BRCA1 and/or MAD2L1 loss of expression was not prognostic, however in a subset of patients treated with vinorelbine, survival was shorter for patients lacking BRCA1/MAD2L1 expression compared with double positive patients (5.9 versus 36.7 months, $p=0.03$ ). Our data implicates BRCA1/MAD2L1 loss as a putative predictive marker of resistance to vinorelbine in mesothelioma, and warrants prospective clinical evaluation. 


\section{Introduction}

Malignant Pleural Mesothelioma (MPM) is an incurable cancer arising from the parietal pleura. Treatment options for mesothelioma remain limited, and personalised therapy is lacking (1). Anti-folate/platinum doublet chemotherapy has been the only licensed treatment since the early 2000s, and there is no FDA or EMA approved second line therapy outside of Japan (2).

The vinca alkaloid spindle poison vinorelbine has demonstrated useful clinical activity in mesothelioma (3-6). We have previously reported that expression of the tumour suppressor breast cancer-associated gene 1 (BRCA1) is an essential regulator of apoptosis induced by vinorelbine in mesothelioma, as evidenced by (i) a positive correlation between BRCA1 expression and in vitro sensitivity; (ii) induction of vinorelbine resistance by BRCA1 targeted RNA interference; (iii) acquired loss of BRCA1 expression in vinorelbine-resistant cells; and (iv) re-sensitisation by ectopic expression of BRCA1 (7). These observations are consistent with other reports $(8,9)$. Loss of detectable BRCA1 protein has been observed in $38 \%$ of mesotheliomas in two independent cohorts of patients, suggesting a potential mechanism of clinical drug resistance (7).

The heterodimeric BRCA1/BARD1 complex modulates mitotic spindle pole assembly, which may contribute to its role in chromosomal stability (10). Loss of BRCA1 has also been reported to inactivate the spindle assembly checkpoint (SAC), causing resistance to the spindle poison paclitaxel (11). The anaphase-promoting complex/cyclosome (APC/C) inhibitor MAD2L1, which is part of the mitotic checkpoint complex, is transcriptionally regulated by BRCA1 via the transcription factor OCT1 (12), implicating a role for BRCA1 in regulating SAC and genome instability. 
On this basis, we hypothesised that the BRCA1/MAD2L1 axis would be an essential regulator of vinorelbine response in mesothelioma in vitro, in ex vivo explants and the clinical context, providing a potential biomarker strategy for selecting patients with mesothelioma that are likely to benefit from vinorelbine treatment, in the relapse setting.

\section{Material and methods}

Reagents and antibodies Vinorelbine (Cat n. V2264) and docetaxel (Cat n. 01885) were obtained by Sigma (Gillingam, UK). Antibodies were used against the following proteins: PARP (Cat n. 9542, Cell Signaling, Hitchin, UK), BRCA1 (Cat n. OP92, clone MS110, Calbiochem), BUBR1 and BRCA2 (Cat n. MAB3612 Clone 8G1, OP95 Clone 2b, Millipore), MAD2L1 (Cat n. sc-47747 Clone 17D10, Santa Cruz, Wembley, UK), and alpha tubulin (Cat n.ab4074, Abcam, Cambridge, UK). Secondary antibodies were anti-mouse IgG, HRP-linked antibody and anti-rabbit IgG, HRPlinked antibody (Cat n. 7076 and 7074, Cell Signaling, Hitchin, UK)

Cell lines MSTO-211H were purchased from ATCC (Cat n. CRL-2081, Middlesex, UK). REN cells were obtained were kindly provided by Dr. S.M. Albelda (University of Pennsylvania, Philadelphia, USA). MSTO cells were grown in RPMI Medium 1640 (Cat n. 31870, Fisher scientific), Glutamax (Cat n. 11574466, Fisher Scientific) and 10\% Foetal Bovine Serum (FBS) (Cat n. F9665 Sigma). REN were grown in Nutrient mixture F12 Ham, Glutamax and 10\% FBS. Vinorelbine-resistant cells (MVR and RVR) were generated by increasing exposure to vinorelbine in a stepwise manner over duration of three months as previously described (7). As apoptosis was induced in the vast majority of cells in the early stage of onset of resistance it is not possible to determine whether a subclonal expansion accounted for the evolution of the SAC 
or whether there was a drug-induced alteration in the phenotype (genetic or epigenetic).

Measurement of apoptosis 5000 cells per well were seeded in 96 wells plate for the Caspase-3 luminescence assay. Vinorelbine in vitro IC50 for REN and MSTO$211 \mathrm{H}$ at 48 hours was previously determined (7). Cells were left untreated or incubated with 50nM (REN) or 100 nM (MSTO-211H) vinorelbine or $20 \mathrm{nM}$ docetaxel. 48 hours following treatment, cells were analysed using the Caspase-Glo 3/7 Assay (Cat n. G8092, Promega, Southampton, UK).

Protein extraction and immunoblotting 48 hours after treatment cells were lysed in RIPA buffer containing protease inhibitors (Cat n. 11836153001, Roche, Burgess Hill UK) and whole-cell lysates were clarified by centrifugation. $40 \mu \mathrm{g}$ of total cell lysates were loaded and separated on SDS-PAGE denaturing gels, transferred to nitrocellulose membranes, and blocked in 5\% milk-PBS-0.1\% tween. Membranes were probed with primary antibodies diluted in $5 \%$ milk-PBS- $0.1 \%$ Tween-20 at $4^{\circ} \mathrm{C}$ overnight. Signal detection was performed with ECL-plus chemiluminescent system (Cat n. GERPN2236, GE Healthcare). Quantifications for western blots images are provided in Supplementary Table 1.

siRNA transfections SiRNAs were obtained from and Qiagen (Hs_BRCA1_13 FlexiTube siRNA, SI02654575, and Hs_MAD2L1_8 FlexiTube siRNA MAD2L1 SI02653847, Manchester, UK). On-Target plus MAD2L1 siRNA was obtained from Dharmacon (Cat n. J003271-13). siRNA transfections (20 nM) were performed using the RNAiMAX transfection reagent (Cat n. 13778075, Invitrogen, Paisley, UK) according to manufacturer's instructions.

Real time quantitative RT-PCR Total RNA was extracted using Trizol (Cat n. 15596026, Invitrogen, Paisley, UK) according to manufacturer's instructions. 
Reverse transcription was performed with High Capacity RNA-to-cDNA Kit (Cat n. 4374966, Applied Biosystem, Paisley, UK). Real-Time PCR was carried out using Power SYBR® Green PCR Master Mix (Cat n. 4368577, Applied Biosystem) after 24 hours of silencing or 48 hours of treatment. QuantiTect primer assays (Qiagen, Manchester, UK) were used for BRCA1 (Cat n. QT00039305), MAD2L1 (Cat n. QT00205709) and actin (Cat n. QT00095451).

Fixed and Live Cell Microscopy Cells were treated with vinorelbine 20nM (this concentration only induced $20 \%$ death in both cell lines but was sufficient to induce mitotic arrest and to allow better interpretation of mitotic arrest dynamics). Cells grown on acid-etched glass coverslips were fixed by incubation in $3.7 \%$ formaldehyde for 10 min followed by permeabilisation in $0.5 \%$ Triton X-100 for 5 min. Cells were blocked in PBS supplemented with 3\% BSA before incubation with the appropriate antibody diluted as required in PBS supplemented with $3 \%$ BSA.

The primary antibody was mouse BUBR1 $(1 \mu \mathrm{g} / \mathrm{ml})$ and the secondary antibodies was Alexa Fluor 488 goat anti-mouse lgGs (1 $\mu \mathrm{g} / \mathrm{ml}$; Cat n. A-11001, Invitrogen). Imaging was performed on a confocal microscope (TCS SP5; Leica) equipped with an inverted microscope (DMI6000 B; Leica) using a 63× oil objective (NA 1.4). Zstacks comprising 30-50 $0.3-\mu \mathrm{m}$ sections were acquired using LAS-AF software (Leica), and deconvolution of 3D image stacks was performed using Huygens software (Scientific Volume Imaging). To quantify BUBR1 intensity, the mean pixel intensity of individual BUBR1 antibody spots was measured and cytoplasmic background subtracted. A minimum of 15 discrete spots was measured for each cell and the mean of these values calculated. Intensities were scaled so that control intensity was $100 \%$. Time-lapse imaging was performed on a Nikon eclipse Ti microscope equipped with an Andor iXonEM+ EMCCD DU 885 camera using a 10x 
phase objective. Cells were cultured in multi-well plates and maintained on a stage with a heated incubator at $37^{\circ} \mathrm{C}$ supplemented with $5 \% \mathrm{CO}_{2}$ using a microscope temperature control system (Life Imaging Services). Images were acquired every 15 min for $24 \mathrm{~h}$ using NIS-elements software. Videos were prepared using ImageJ (National Institutes of Health).

Cell cycle analysis Cells were treated with vinorelbine 20nM (this concentration only induced $20 \%$ death in both cell lines but was sufficient to induce mitotic arrest). Cell cycle distribution was analysed by flow cytometry on a FACSCanto II (Becton Dickinson). Cells were collected, washed with PBS and fixed in $70 \%$ ice-cold ethanol for $1 \mathrm{~h}$ or overnight before being stained with propidium iodide (PI) (Cat $\mathrm{n}$. P4864, Sigma) in PBS containing RNase A (Cat n. R6513, Sigma).

Primary Mesothelioma Explants Twenty patients with histologically confirmed epithelioid malignant pleural mesothelioma scheduled to undergo extended pleurectomy and decortication were consented to provide research samples under ethics approval 14/LO/1527. Informed consent to provide research samples was obtained from all patients. Patient characteristics are described in Supplementary table 2. All primary pleural tissue was sectioned into fragments measuring approximately $8 \mathrm{~mm}^{3}$. Tissue explants were cultured in RPMI Medium 1640, 1\% Glutamax, 10\% Foetal Bovine Serum (FBS), 1\% Penicillin/Streptomycin and 1\% fungizone. Explants were allowed to recover overnight prior treatment. Live explants were treated with vinorelbine $1 \mu \mathrm{M}$ for 72 hours. This concentration is about 10 times in vitro average IC50) taking into account intra-patient heterogenicity, tumour microenvironment and lower sensitivity compared to $2 \mathrm{D}$ models. This concentration is clinically relevant and comparable to the maximum plasma concentration of 
vinorelbine in clinical studies. After fixation and embedding, $5 \mu \mathrm{m}$ sections were used for immunohistochemistry, as previously described (13).

Archival tissue samples A total of 48 formalin fixed paraffin embedded mesothelioma tissue samples were collected from 3 centres (UCL ethics approval 06/Q0505/12; Mayo Clinic ethics approval 13-005053; NKI ethics approval N12PRO, N14PLU), enabling the assessment of BRCA1 and MAD2L1 expression and correlation with clinical outcome. Patient characteristics are described in Supplementary table 3.

Immunohistochemistry Primary antibodies were diluted in 1\% goat serum/0.1\% BSA/PBS (MAD2L1, Santacruz, 1:50 Cat n. sc-374131, Clone C-10; cleaved PARP, Abcam 1:6000 Cat n. ab4830; BRCA1, Calbiochem, 1:100 Cat n. Cat n. OP92, clone MS110). All antibodies were incubated at $4^{\circ} \mathrm{C}$ overnight. The Novolink Polymer Detection Kit (Cat n. RE7150-CE, Leica, Milton Keynes, UK) was used according to the manufacturer's instructions. Sections were counterstained with hematoxylin and mounted using Leica Sub-X mounting medium (Leica). Images were taken on a Hamamatsu Nanozoomer Digital slide scanner. For BRCA1, automated staining has been performed on the Leica Bond III platform and the Leica Bond Polymer Refine DAB was used for detection.

The scoring was carried out by a pathologist with orthogonal validation using image process by Qupath (14). BRCA1 positive tumours were defined as those demonstrating $>10 \%$ of cells with nuclear staining (7). MAD2L1 positive tumours were defined as those demonstrating $>10 \%$ of cells with either nuclear or cytoplasmic staining (15). Immunohistochemical staining of cleaved poly ADP-ribose polymerase, was scored as percentage of cells with nuclear staining. 
Statistical Analysis Dose-response curves were fitted using non-linear regression (GraphPad Prism version 6.0, GraphPad Software, LaJolla, CA, USA). The significance of the data has been assessed with t-test (two tails), Wilcoxon test, Mann Whitney or one-way Anova (Tukey's multiple comparisons test). For KaplanMeier curves the log-rank (Mantel Cox) test was applied. All bar charts show means $-/+$ SD. Results are from at least 3 biological replicates. All $p$ values less than 0.05 were considered significant.

\section{RESULTS}

BRCA1 is essential for induction of SAC activation and apoptosis by vinorelbine

To directly test whether or not BRCA1 expression is required for SAC activation and consequent apoptosis induced by vinorelbine in mesothelioma, we assessed the effect of depletion of BRCA1 by RNA interference in MSTO-211H and REN mesothelioma cells. Time-lapse imaging after treatment with vinorelbine showed that while parental cells progressed rapidly through mitosis in the absence of vinorelbine, cells treated with vinorelbine rounded up in a manner indicative of mitotic entry and remained in this state for an extended period of time. In contrast, BRCA1-depleted cells progressed through mitosis in the presence of vinorelbine indicating a defective SAC (Figure 1A). Cell cycle analysis revealed that 24 hours after vinorelbine treatment, at least $70 \%$ of control cells (siNT) were present in the G2/M fraction as compared to $\sim 20 \%$ in the absence of vinorelbine, whereas there was no significant accumulation of cells at G2/M in response to vinorelbine following knockdown of BRCA1, consistent with inactivation of the SAC (Figure 1B). 
To assess the functional effects of BRCA1 depletion on the SAC in response to vinorelbine, we analyzed the localisation of the SAC component, BUBR1 which localizes to kinetochores in the context of a functional checkpoint. Upon vinorelbine treatment of BRCA1 positive cells, BUBR1 exhibited strong staining of kinetochores, indicative of an active SAC. However, in BRCA1 silenced cells the intensity of BUBR1 was significantly reduced at kinetochores (Figure 1C). Total cellular BUBR1 protein expression was increased following BRCA1 silencing in MSTO-211H, although this difference was not significant in REN cells (Figure 1D). Vinorelbine induced apoptosis in control cells (siNT) as shown by a significant increase in caspase 3 activity compared to untreated control, whilst BRCA1, but not BRCA2 depletion (Supplementary Figure 1), caused a significant reduction in vinorelbineinduced apoptosis (Figure 1E).

\section{MAD2L1 silencing phenocopies BRCA1 loss, preventing vinorelbine-induced}

\section{cell death}

MAD2L1 has been identified as a transcriptional target of BRCA1 (12). Accordingly, following BRCA1 silencing by RNA interference, we observed downregulation of MAD2L1 at both the mRNA (Figure 2A) and protein levels in MSTO-211H and REN cells (Figure 2B).

To confirm that BRCA1 mediates the response to vinorelbine through regulation of the SAC via MAD2L1 we determined the effects of MAD2L1 depletion on vinorelbine-induced apoptosis. Treatment with vinorelbine in MSTO-211H and REN control cells (siNT) induced significant activation of caspase 3 compared to untreated control. MAD2L1 depletion by two different siRNAs phenocopied BRCA1 silencing and rescued cells from vinorelbine-induced apoptosis, confirming that the 
BRCA1/MAD2L1 axis is required to mediate the proapoptotic response to vinorelbine (Figure 2C).

\section{Vinorelbine-resistant mesothelioma cells acquire SAC deficiency and down-} regulate MAD2L1

To determine if cells under selection for resistance to vinorelbine might acquire SAC deficiency through loss of BRCA1 and MAD2L1, we studied MVR and RVR cell lines which had been selected for resistance to vinorelbine (7). MVR and RVR cells expressed significantly lower constitutive levels of BRCA1 protein compared to the parental cells, with a reduction in MAD2L1 mRNA and protein expression in MVR and RVR compared to parental cells (Figure 3A).

Consistent with BRCA1 RNA interference experiments, resistant cells showed a significant reduction in caspase 3 activity in response to vinorelbine, compared to parental cell lines (Figure 3B). Upon treatment with vinorelbine, parental REN cells rounded up in a manner indicative of mitotic arrest, while RVR cells progressed through mitosis consistent with SAC deficiency. A short delay in mitotic progression upon vinorelbine treatment was observed by live cell imaging in MVR cells although, in contrast to MSTO-211H cells, MVR cells rapidly resumed cell cycle progression following the transient mitotic arrest (Figure 3C). Resistant cells showed a reduction in the G2/M population by flow cytometry after treatment with vinorelbine (Figure 3D).

Localisation of BUBR1 to mitotic kinetochores was reduced by approximately $80 \%$ in RVR compared to parental REN cells upon vinorelbine treatment. There was a more modest, albeit significant reduction in BUBR1 intensity $(\sim 50 \%)$ in MVR versus MSTO-211H parental mitotic cells (Figure 3E). This supports the hypothesis that the 
short delay in mitotic progression observed upon vinorelbine treatment of MVR cells is secondary to acquisition of a defective SAC. An increase in protein levels of BUBR1 in MVR compared to MSTO-211H was observed, although there was no change in expression upon treatment with vinorelbine in either cell line. In contrast, basal levels of BUBR1 were not different between REN and RVR. Vinorelbine induced a decrease in BUBR1 expression in both parental and resistant cells (Figure 3F). To establish if this acquired SAC defect would confer resistance to other spindle poisons, the microtubule stabiliser, docetaxel was studied. Apoptosis in response to docetaxel was significantly reduced following BRCA1 silencing in parental MSTO$211 \mathrm{H}$ and REN cells (Supplementary figure 2A) and in vinorelbine-resistant MRV and RVR cells (Supplementary figure 2B). This was associated with loss of SACmediated mitotic arrest (Supplementary figure 2C).

\section{Mesothelioma explants with reduced BRCA1/MAD2L1 expression exhibit}

\section{vinorelbine resistance}

Live mesothelioma explants cultures were generated from a cohort of 20 patients. BRCA1 positive staining was observed in $85 \%$ of samples $(n=17)$. BRCA1 negativity was observed in $15 \%$ of samples $(n=3)$ and was associated with MAD2L1 negativity in 1 of these samples. Following ex-vivo treatment with vinorelbine, nuclear cleaved PARP staining, indicative of apoptosis, was observed only in the context of BRCA1 positivity, in $59 \%$ of cases $(n=10)$, while all cases negative for either BRCA1 or MAD2L1 $(n=3)$ were resistant to vinorelbine with a $0 \%$ response rate (Figure $4 A, B$ ). ROC curves for BRCA1 and MAD2L1 showed a promising fit, although the curves were not significant probably due to the sample size $(\mathrm{BRCA} 1 \mathrm{AUC}=0.853, \mathrm{p}=$ 
0.057 (95\% Cl 0.677-1.0), MAD2L1 AUC = 0.792, $p=0.186(95 \% \mathrm{Cl} 0.568-1.0))$. (Supplementary Figure 3) .

This data is consistent with BRCA1/MAD2L1 regulated SAC as a mediator of response to vinorelbine.

\section{Loss of BRCA1 or MAD2L1 expression correlates with poorer survival following vinorelbine treatment}

In a cohort of 48 patients, BRCA1 protein expression was lost in $39.6 \%$ of patients $(n=19)$, of which $68.4 \%(n=13)$ also lacked MAD2L1 expression (Figure 4C). BRCA1 and MAD2L1 were not prognostic in this cohort (Figure 4D, 4E, Supplementary Figure 4).

To explore the impact of BRCA1/MAD2L1 expression on clinical outcome following vinorelbine treatment in the relapse setting, patients who had received second-line treatment with vinorelbine were stratified by BRCA1 and MAD2L1 protein expression $(n=10)$. BRCA1/MAD2L1 negative patients $(n=6)$ had a worse median overall survival of 5.9 months versus 36.7 months for BRCA1/MAD2L1 positive patients $(n=4),(p=0.03$, hazard ratio $=3.737)$, consistent with resistance to vinorelbine (Figure 4F). The effect of MAD2L1 negativity only $(n=3)$ on survival was not significant $(p=0.27)$, although this is likely to be due to the very small sample size (Supplementary Figure 5).

\section{DISCUSSION}

Despite recent advances in our understanding of inter-patient genomic heterogeneity in mesothelioma, targeted therapies are lacking, and no treatment has yet demonstrated an improvement in survival in the relapsed setting. The spindle poison 
vinorelbine mediates microtubule depolymerisation and has demonstrated clinically useful activity in mesothelioma with a reported disease control rate of $68 \%$ in the relapsed setting $(3,5,6)$. There are at present no validated predictive biomarkers in use to facilitate patient stratification for this agent.

Mesotheliomas exhibit a high degree of aneuploidy and genomic instability (16). The fidelity of the SAC is essential for maintaining genome stability (17), and SAC deregulation has been previously reported in mesothelioma (18). Consistent with our results, sensitivity to the spindle poison paclitaxel has been reported to be dependent on an intact SAC as evidenced by functional genetic studies involving suppression of MAD2L1, BUBR1 (19) or BRCA1 (11) in breast cancer, whereby silencing of BRCA1 induced >1000 fold cross-resistance to multiple spindle poisons in breast cancer cell lines (8).

BRCA1 has been reported to regulate the response to spindle poisons by affecting the dynamics of SAC activation and mitotic arrest $(11,20)$. The heterodimeric BRCA1/BARD1 complex mediates E3 ubiquitin ligase activity, and has been shown to transactivate the anaphase-promoting complex APC/C inhibitor MAD2L1 via the Oct1 transcription factor (12). Several studies have uncovered an array of BRCA1 targets involved in the maintenance of chromosome integrity during cell cycle (2123), and a number of factors are likely involved in regulating the spindle assembly checkpoint. However the specific clinical relevance of these factors in mesothelioma needs to be addressed. Activation of $\mathrm{p} 21$ and $\mathrm{p} 27$ by BRCA1 has been shown to lead to arrest cells at the G1/S phase. Transcriptional repression of cyclin B by BRCA1 is involved in mitotic entry and lack of BRCA1 has been shown to prevent a G2/M arrest in response to ionizing radiation. The protein expression of Cyclin B has been explored in our model, although no significant change in basal expression was 
observed between parental and resistant cells or after silencing of BRCA1 (Supplementary Figure 6). Finally, BRCA1-mediated transactivation of GADD45 was shown to be important in the regulation of the mitotic checkpoint, through the regulation of the CyclinB-cdc2 complex (22).

Understanding of the precise mechanism by which BRCA1 regulates cell fate following mitotic arrest induced by vinorelbine has remained elusive. Here we show that vinorelbine-induced apoptosis occurs as a result of prolonged activation of the SAC with subsequent mitotic catastrophe that is BRCA1 dependent. Furthermore, we have shown that vinorelbine resistant cells evolve a dysfunctional SAC associated with downregulation of both BRCA1 and MAD2L1.

The basis for loss of BRCA1 expression in mesothelioma is unknown. In contrast to breast and ovarian cancers, mesotheliomas have not been reported to harbour biallelic somatic alteration of BRCA1 or MAD2L1 $(24,25)$. BRCA1-associated protein 1 (BAP1) is one of the most commonly mutated genes in mesothelioma (24-27). Deletion of BAP1 has been reported to reduce BRCA1 expression, which can be rescued with ectopic expression of BAP1. This implicates possible control of BRCA1 stability and therefore SAC by BAP1 (28). We have observed a positive correlation between BAP1 expression, SAC activation and consequent sensitivity to vinorelbine and Anetumab Ravtansine. Also primary cell lines harbouring BAP1 mutation showed reduced BRCA1/MAD2 protein expression (29). This putative association between BAP1 and SAC regulation deserves further exploration. Kumar and colleagues recently reported that BAP1 expression correlates with vinorelbine sensitivity in the MS01 trial cohort (22).

From a translational perspective, loss of BRCA1 and/or MAD2L1 expression in mesothelioma may have implications for predicting the clinical response in patients 
treated with vinorelbine in the relapse setting. Here we show that BRCA1 is lost in $39.6 \%$ of cases in a cohort of 48 patients of which $68.4 \%$ of BRCA1 negative tumours also lacked expression of MAD2L1 (i.e. double negative). In patients treated with vinorelbine second line, BRCA1/MAD2L loss predicted clinical outcome suggesting possible utility as a biomarker.

These findings will be explored in an independent validation associated with the Vinorelbine in Mesothelioma (VIM) clinical trial (NCT2139904), a large open label randomised controlled phase II trial (second line treatment with oral vinorelbine versus active symptom control). The analysis of the VIM trial will allow testing for both prognostic and predictive effects of BRCA1 and MAD2L1 either alone or with combined loss.

\section{Authors' Contributions}

Conception and design: S. Busacca, D.A. Fennell, A. M. Fry

Acquisition of data: S. Busacca, L. O' Regan, A. Singh, J. Dzialo, A. J. Sharkey, A. G. Dawson, N. Kumar, L. M. Schunselaar, N. Guppy, A. Nakas, A. S. Mansfield, S. M. Janes, P. Baas

Analysis and interpretation of data: S. Busacca, M. Sheaff, D.A. Fennell, A. M. Fry Writing, review, and/or revision of the manuscript: All authors

Funding: SB was funded by The June Hancock Mesothelioma research fund (JH/09/2011).

\section{REFERENCES}

1. Yap TA, Aerts JG, Popat S, Fennell DA. Novel insights into mesothelioma biology and implications for therapy. Nat Rev Cancer 2017;17(8):475-88 doi 10.1038/nrc.2017.42. 
Author Manuscript Published OnlineFirst on November 6, 2020; DOI: 10.1158/1535-7163.MCT-20-0363

Author manuscripts have been peer reviewed and accepted for publication but have not yet been edited.

2. Vogelzang NJ, Rusthoven JJ, Symanowski J, Denham C, Kaukel E, Ruffie P, et al. Phase III study of pemetrexed in combination with cisplatin versus cisplatin alone in patients with malignant pleural mesothelioma. J Clin Oncol 2003;21(14):2636-44 doi 10.1200/JCO.2003.11.136

JCO.2003.11.136 [pii].

3. Steele JP, Shamash J, Evans MT, Gower NH, Tischkowitz MD, Rudd RM. Phase II study of vinorelbine in patients with malignant pleural mesothelioma. J Clin Oncol 2000;18(23):3912-7.

4. $\quad$ Fennell DA, JP CS, Shamash J, Sheaff MT, Evans MT, Goonewardene TI, et al. Phase II trial of vinorelbine and oxaliplatin as first-line therapy in malignant pleural mesothelioma. Lung Cancer 2005;47(2):277-81 doi 10.1016/j.lungcan.2004.08.005.

5. Muers MF, Stephens RJ, Fisher P, Darlison L, Higgs CM, Lowry E, et al. Active symptom control with or without chemotherapy in the treatment of patients with malignant pleural mesothelioma (MS01): a multicentre randomised trial. Lancet 2008;371(9625):1685-94 doi 10.1016/S01406736(08)60727-8.

6. Stebbing J, Powles T, McPherson K, Shamash J, Wells P, Sheaff MT, et al. The efficacy and safety of weekly vinorelbine in relapsed malignant pleural mesothelioma. Lung Cancer 2009;63(1):94-7 doi S0169-5002(08)00194-3 [pii] 10.1016/j.lungcan.2008.04.001.

7. Busacca S, Sheaff M, Arthur K, Gray SG, O'Byrne KJ, Richard DJ, et al. BRCA1 is an essential mediator of vinorelbine-induced apoptosis in mesothelioma. The Journal of pathology 2012;227(2):200-8 doi 10.1002/path.3979.

8. Quinn JE, Kennedy RD, Mullan PB, Gilmore PM, Carty M, Johnston PG, et al. BRCA1 functions as a differential modulator of chemotherapy-induced apoptosis. Cancer Res 2003;63(19):6221-8.

9. Mullan PB, Quinn JE, Gilmore PM, McWilliams S, Andrews H, Gervin C, et al. BRCA1 and GADD45 mediated G2/M cell cycle arrest in response to antimicrotubule agents. Oncogene 2001;20(43):6123-31 doi 10.1038/sj.onc.1204712.

10. Joukov V, Groen AC, Prokhorova T, Gerson R, White E, Rodriguez A, et al. The BRCA1/BARD1 heterodimer modulates ran-dependent mitotic spindle assembly. Cell 2006;127(3):539-52 doi 10.1016/j.cell.2006.08.053.

11. Chabalier C, Lamare C, Racca C, Privat M, Valette A, Larminat F. BRCA1 downregulation leads to premature inactivation of spindle checkpoint and confers paclitaxel resistance. Cell cycle 2006;5(9):1001-7.

12. Wang $\mathrm{RH}, \mathrm{Yu} \mathrm{H}$, Deng $\mathrm{CX}$. A requirement for breast-cancer-associated gene 1 (BRCA1) in the spindle checkpoint. Proceedings of the National Academy of Sciences of the United States of America 2004;101(49):17108-13 doi 10.1073/pnas.0407585101.

13. Busacca S, Law EW, Powley IR, Proia DA, Sequeira M, Le Quesne J, et al. Resistance to HSP90 inhibition involving loss of MCL1 addiction. Oncogene 2016;35(12):1483-92 doi 10.1038/onc.2015.213.

14. Bankhead P, Loughrey MB, Fernandez JA, Dombrowski Y, McArt DG, Dunne PD, et al. QuPath: Open source software for digital pathology image analysis. Sci Rep 2017;7(1):16878 doi 10.1038/s41598-017-17204-5.

15. Wang L, Yin F, Du Y, Du W, Chen B, Zhang Y, et al. MAD2 as a key component of mitotic checkpoint: A probable prognostic factor for gastric 
Author Manuscript Published OnlineFirst on November 6, 2020; DOI: 10.1158/1535-7163.MCT-20-0363

Author manuscripts have been peer reviewed and accepted for publication but have not yet been edited.

cancer. Am J Clin Pathol 2009;131(6):793-801 doi 10.1309/AJCPBMHHDOHFCY8W.

16. Isobe $H$, Sridhar KS, Doria R, Cohen F, Raub WA, Saldana $M$, et al. Prognostic significance of DNA aneuploidy in diffuse malignant mesothelioma. Cytometry 1995;19(1):86-91 doi 10.1002/cyto.990190111.

17. Xu X, Weaver Z, Linke SP, Li C, Gotay J, Wang XW, et al. Centrosome amplification and a defective G2-M cell cycle checkpoint induce genetic instability in BRCA1 exon 11 isoform-deficient cells. Mol Cell 1999;3(3):38995.

18. Suraokar MB, Nunez MI, Diao L, Chow CW, Kim D, Behrens C, et al. Expression profiling stratifies mesothelioma tumors and signifies deregulation of spindle checkpoint pathway and microtubule network with therapeutic implications. Annals of oncology : official journal of the European Society for Medical Oncology / ESMO 2014;25(6):1184-92 doi 10.1093/annonc/mdu127.

19. Sudo T, Nitta M, Saya H, Ueno NT. Dependence of paclitaxel sensitivity on a functional spindle assembly checkpoint. Cancer research 2004;64(7):2502-8.

20. Sung $M$, Giannakakou P. BRCA1 regulates microtubule dynamics and taxane-induced apoptotic cell signaling. Oncogene 2014;33(11):1418-28 doi 10.1038/onc.2013.85.

21. Gorski JJ, Savage KI, Mulligan JM, McDade SS, Blayney JK, Ge Z, et al. Profiling of the BRCA1 transcriptome through microarray and ChIP-chip analysis. Nucleic Acids Res 2011;39(22):9536-48 doi 10.1093/nar/gkr679.

22. Mullan PB, Quinn JE, Harkin DP. The role of BRCA1 in transcriptional regulation and cell cycle control. Oncogene 2006;25(43):5854-63 doi 10.1038/sj.onc. 1209872.

23. Venkitaraman AR. Cancer suppression by the chromosome custodians, BRCA1 and BRCA2. Science 2014;343(6178):1470-5 doi 10.1126/science.1252230.

24. Bueno R, Stawiski EW, Goldstein LD, Durinck S, De Rienzo A, Modrusan Z, et al. Comprehensive genomic analysis of malignant pleural mesothelioma identifies recurrent mutations, gene fusions and splicing alterations. Nature genetics 2016;48(4):407-16 doi 10.1038/ng.3520.

25. Hmeljak J, Sanchez-Vega F, Hoadley KA, Shih J, Stewart C, Heiman D, et al. Integrative Molecular Characterization of Malignant Pleural Mesothelioma. Cancer Discov 2018;8(12):1548-65 doi 10.1158/2159-8290.CD-18-0804.

26. Testa JR, Cheung M, Pei J, Below JE, Tan Y, Sementino E, et al. Germline BAP1 mutations predispose to malignant mesothelioma. Nat Genet 2011;43(10):1022-5 doi 10.1038/ng.912.

27. Bott M, Brevet M, Taylor BS, Shimizu S, Ito T, Wang L, et al. The nuclear deubiquitinase BAP1 is commonly inactivated by somatic mutations and 3p21.1 losses in malignant pleural mesothelioma. Nature genetics 2011;43(7):668-72 doi 10.1038/ng.855.

28. Hakiri S, Osada $\mathrm{H}$, Ishiguro $\mathrm{F}$, Murakami $\mathrm{H}$, Murakami-Tonami $\mathrm{Y}$, Yokoi $\mathrm{K}$, et al. Functional differences between wild-type and mutant-type BRCA1associated protein 1 tumor suppressor against malignant mesothelioma cells. Cancer Sci 2015;106(8):990-9 doi 10.1111/cas.12698.

29. Singh A, Luo JL, Busacca S, Dawson AG, Kolluri KK, Janes SM, et al. Loss of BAP1/BRCA1 counteracts spindle assembly checkpoint activation by the antimesothelin antibody drug-conjugate anetumab ravtansine 2020. 


\section{Figure legends}

Figure 1. BRCA1 loss prevents vinorelbine-induced cell death through mitotic slippage. A) 24 hours after siRNA transfection, cells were treated with $20 \mathrm{nM}$ vinorelbine and subjected to time-lapse imaging using phase contrast microscopy. Cells were imaged every 15 minutes for $25 \mathrm{~h}$. Control cells were treated with DMSO. Stills from representative movies are shown. Scale bar $200 \mu \mathrm{m}$. Histograms show the time that cells spent in a rounded up state indicative of mitosis. MSTO-211H: siNT NT vs siNT VNB $p<0.0001$; siNT VNB vs siBRCA1 VNB $p<0.0001$. REN: siNT NT vs siNT VNB $p<0.0001$; siNT VNB vs siBRCA1 VNB $p<0.0001$. B) 24 hours after siRNA transfection cells were treated with $20 \mathrm{nM}$ vinorelbine for further 16 hours. Cells were then fixed and analysed by flow cytometry. The histograms indicate the percentage of cells in G2/M based on the flow cytometry plots. MSTO-211H: siNT NT vs siNT VNB $p=0.0045 ;$ siNT VNB vs siBRCA1 VNB $p=0.0044$. REN: siNT NT vs siNT VNB $p=0.0007 ;$ siNT VNB vs siBRCA1 VNB $p=0.0018$. C) 24 hours after siRNA transfection, cells were treated with $20 \mathrm{nM}$ vinorelbine for further 16 hours. Cell were stained with BUBR1 antibody and DNA was stained with Hoechst 33258. Scale bar, $10 \mu \mathrm{m}$. The relative intensity of BUBR1 was measured and is expressed relative to BUBR1 intensity in control cells. MSTO-211H: siNT vs siBRCA1 $p=0.0038$. REN: siNT vs siBRCA1 $p=0.0001$. D) 24 hours after siRNA transfection, cells were treated with $20 \mathrm{nM}$ vinorelbine for further 16 hours. Western blot is showing BUBR1 protein expression and tubulin is used as loading control. Replicate images are shown in Supplementary figure 7. E) 24 hours following transfection, cells were treated with vinorelbine $50 \mathrm{nM}(\mathrm{REN})$ or $100 \mathrm{nM}(\mathrm{MSTO}-211 \mathrm{H})$ for a further 48 hours and caspase 3 activity measured. Data were normalised to untreated siNT control. MSTO-211H: 
siNT NT vs siNT VNB $p=0.0001$; $\operatorname{siBRCA1}$ NT vs siBRCA1 VNB $p=0.0019$; siNT VNB vs siBRCA1 VNB $p=0.0008$. REN: siNT NT vs siNT VNB $p=0.0012 ;$ siNT VNB vs $\operatorname{siBRCA} 1$ VNB $p=0.0057$. Western blots show down-regulation of BRCA1 after silencing.

Figure 2. MAD2L1 downregulation recapitulates loss of BRCA1 and is associated with vinorelbine resistance.

A) BRCA1 and MAD2L1 mRNA expression was evaluated by qRT-PCR on RNA extracted from cells transfected with siNT and siBRCA1 for 24 hours. Data were normalised to untreated control. MSTO-211H: BRCA1 expression siNT vs siBRCA1 $\mathrm{p}<0.0001 ;$ MAD2L1 expression siNT vs siBRCA1 $p<0.0001$. REN: BRCA1 expression siNT vs siBRCA1 $p<0.0001$; MAD2L1 expression siNT vs siBRCA1 $p<0.0001$. B) Western blots show downregulation of BRCA1 and MAD2L1 24 hours after silencing and treatment with vinorelbine 50nM (REN) or $100 \mathrm{nM}(\mathrm{MSTO}-211 \mathrm{H})$ for 48 hours. Replicate images are shown in Supplementary figure 7. C) MSTO and REN cells were transfected with siNT and siMAD2L1 (sequence \#1 from Qiagen and sequence \#2 from Dharmacon). 24 hours following transfection, cells were treated with vinorelbine $50 \mathrm{nM}$ (REN) or $100 \mathrm{nM}(\mathrm{MSTO}-211 \mathrm{H})$ for a further 48 hours and caspase 3 activity measured. Data were normalised to untreated siNT control. Western blots show downregulation of MAD2L1 24 hours after silencing and treatment with vinorelbine $100 \mathrm{nM}$ for 48 hours. MSTO-211H: siNT NT vs siNT VNB $\mathrm{p}<0.0001$; siNT VNB vs siMAD2L1 VNB $p<0.0001$ for both siRNAs. REN: siNT NT vs siNT VNB $p<0.0001$; siNT VNB vs siMAD2L1VNB $p<0.0001$ for both siRNAs for both siRNAs. 
Author Manuscript Published OnlineFirst on November 6, 2020; DOI: 10.1158/1535-7163.MCT-20-0363

Author manuscripts have been peer reviewed and accepted for publication but have not yet been edited.

Figure 3. Selection of resistance to vinorelbine recapitulates downregulation of

\section{BRCA1.}

A) BRCA1 and MAD2L1 mRNA expression was evaluated by qRT-PCR on RNA extracted from parental and resistant cells. Data were normalised to untreated control. Western blots show BRCA1 and MAD2L1 protein before and after treatment with vinorelbine $100 \mathrm{nM}$ for 48 hours. BRCA1 expression: MSTO-211H vs MVR $p<0.0001 ;$ REN vs RVR $p<0.0001$. MAD2L1 expression: MSTO-211H vs MVR $p=0.0030 ;$ REN vs RVR $p=0.0025$. B) Cells were treated with vinorelbine 50nM (REN) or $100 \mathrm{nM}(\mathrm{MSTO}-211 \mathrm{H})$ for 48 hours and caspase 3 activity measured. Data were normalised to parental cells. MSTO-211H NT vs MSTO-211H VNB $p<0.0001$; MSTO211H VNB vs MVR VNB $p<0.0001 ;$ MVR NT vs MVR VNB $p=0.0255$. REN NT vs REN VNB $p<0.0001$; REN VNB vs RVR VNB $p<0.0001$. C) Cells were treated with $20 \mathrm{nM}$ vinorelbine before time-lapse imaging using phase contrast microscopy. Cells were imaged every 15 mins for $25 \mathrm{~h}$ following addition of drug. Control cells were treated with DMSO. Stills from representative movies are shown. Scale bar $200 \mu \mathrm{m}$. Times that cells spent in a rounded up state indicative of mitosis was measured and is shown in the histograms. MSTO-211H NT vs MSTO-211H VNB $\mathrm{p}<0.0001 ; \mathrm{MSTO} 211 \mathrm{H}$ VNB vs MVR VNB $\mathrm{p}<0.0001 ;$ MVR NT vs MVR VNB $p<0.0001$. REN NT vs REN VNB $p<0.0001$; REN VNB vs RVR VNB $p<0.0001$. D) Cells were treated with $20 \mathrm{nM}$ vinorelbine for 16 hours. Cells were then fixed and analysed by flow cytometry. The histograms indicate the percentage of cells in G2/M based on the flow cytometry plots. . MSTO-211H NT vs MSTO-211H VNB $p<0.0001$; MSTO211H VNB vs MVR VNB $p=0.0021 ;$ MVR NT vs MVR VNB $p<0.0001$. REN NT vs REN VNB $p=0.0092 ; \operatorname{REN}$ VNB vs RVR VNB $p=0.0003$. E) Cells were treated with $20 \mathrm{nM}$ vinorelbine for 16 hours. Cell were stained with BUBR1 antibody and 
DNA was stained with Hoechst 33258. Scale bar, $10 \mu \mathrm{m}$. The relative intensity of BUBR1 was measured and is expressed relative to BUBR1 intensity in control cells. MSTO211H vs MVR $p=0.0002 ;$ REN vs RVR $p<0.0001$. F) Cells were treated with $20 \mathrm{nM}$ vinorelbine for 16 hours. Western blot is showing BUBR1 protein expression and tubulin is used as loading control Replicate images are shown in Supplementary figure 7.

Figure 4. BRCA1 and MAD2L1 downregulation is associated with resistance to vinorelbine in mesothelioma (A) Dot plot showing response in mesothelioma explants treated with vinorelbine $1 \mu \mathrm{M}$ (median), measured as \% of nuclear cleaved PARP staining. Baseline for response was set as 10\% nuclear cleaved PARP. (B) Immunohistochemistry showing BRCA1 and MAD2L1 expression and PARP cleavage in explants. Data are shown for the best responder, positive for BRCA1 and MAD2L1, and a non-responder, negative for BRCA1 and MAD2L1. Images are shown at 5x and 40x magnification. C) Representative images of BRCA1 and MAD2L1 positive and negative tumour samples. Images are shown at $5 x$ and 40x magnification. D) Kaplan Meier curve for overall survival (OS) in patients $(n=48)$ stratified according to BRCA1 expression E) Kaplan Meier curve for overall survival (OS) in patients ( $\mathrm{n}=48$ ) stratified according to MAD2L1 expression. F) Kaplan Meier curves for overall survival (OS) in patients treated with vinorelbine second line $(n=10)$ $(p=0.03$, hazard ratio $=3.737)$. 


\section{Figure 1}

A

Hours 1

10

15

20

25
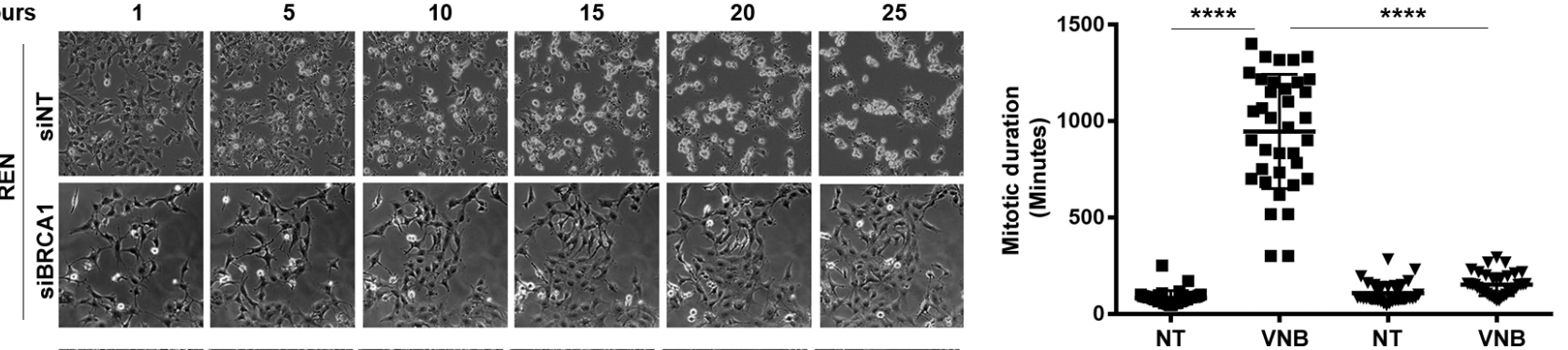

- siNT

$\checkmark$ siBRCA1
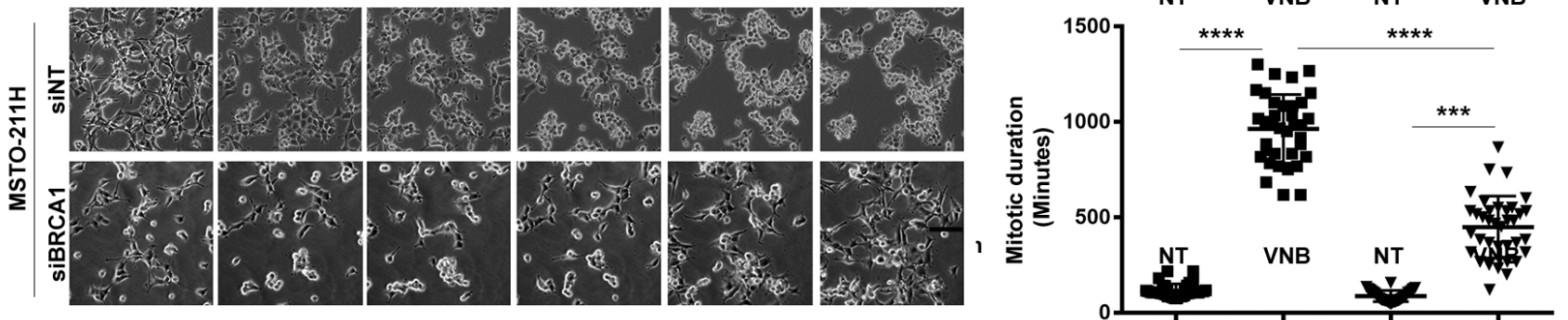

B
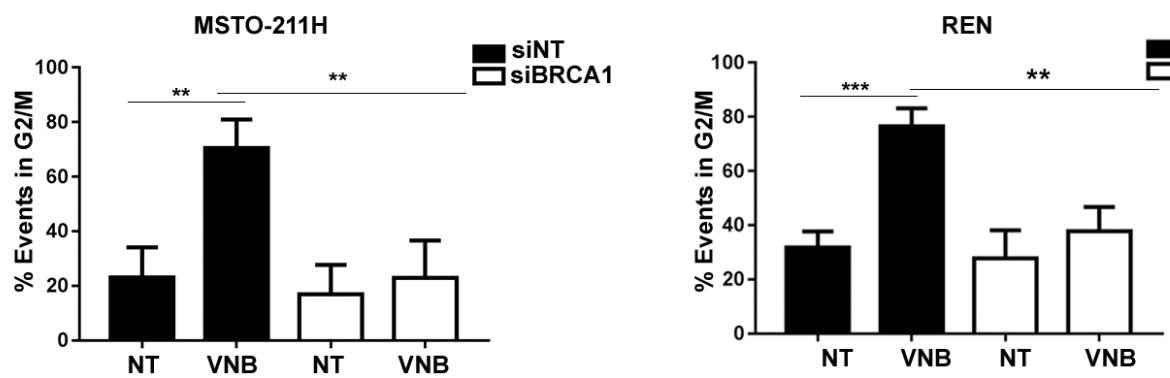

C
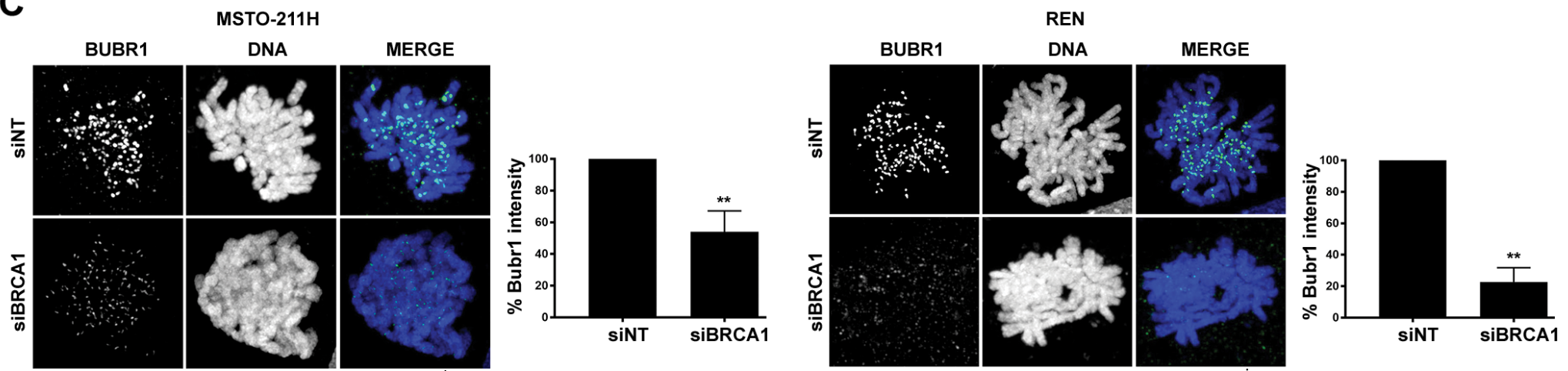

D

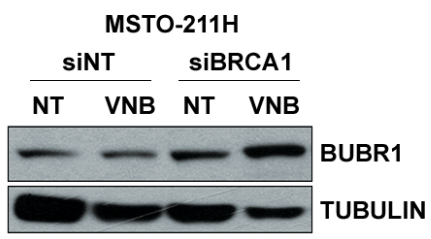

REN
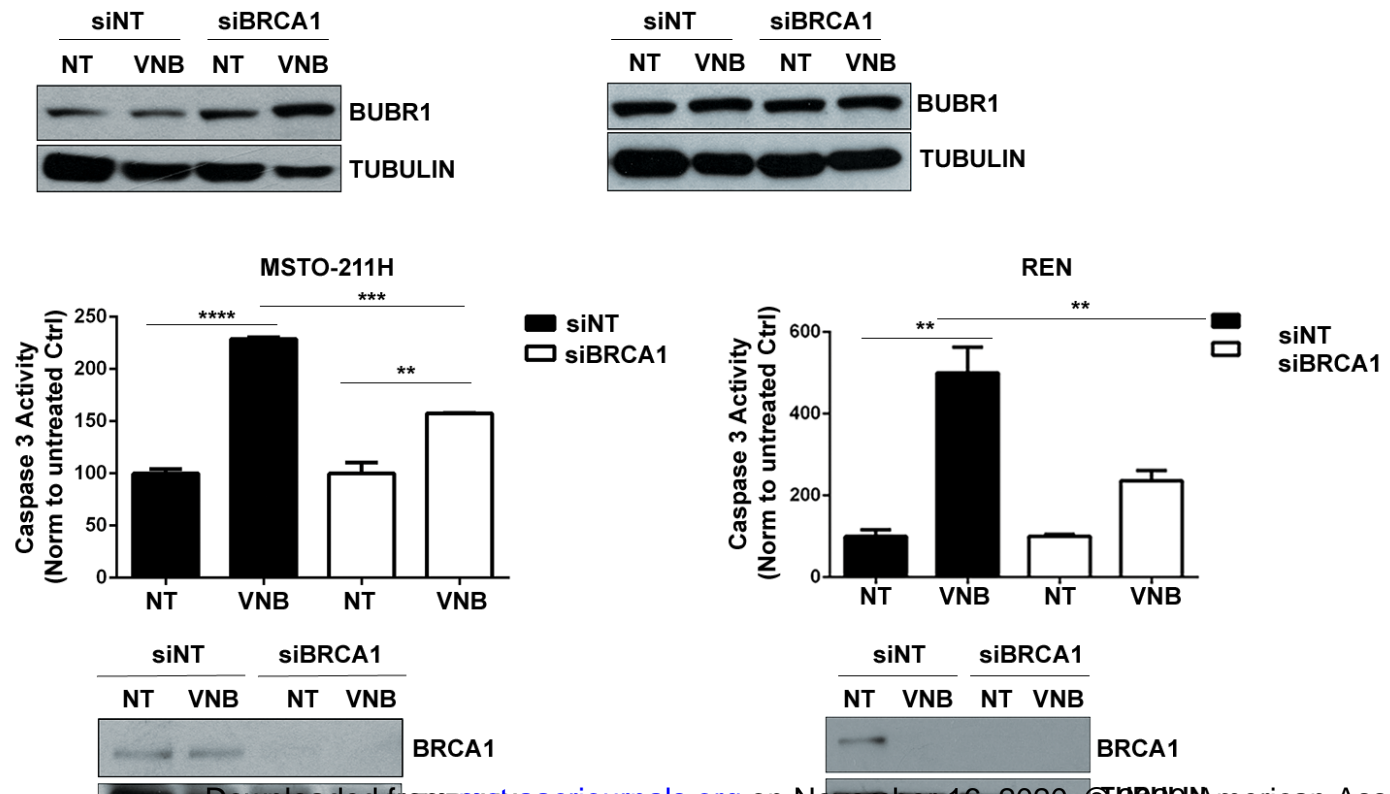

$\frac{\text { SINT }}{\text { NT VNB }} \stackrel{\text { SIBRCA1 }}{\text { NT VNB }}$
BRCA1

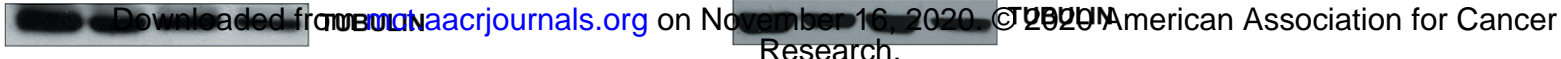




\section{Figure 2}

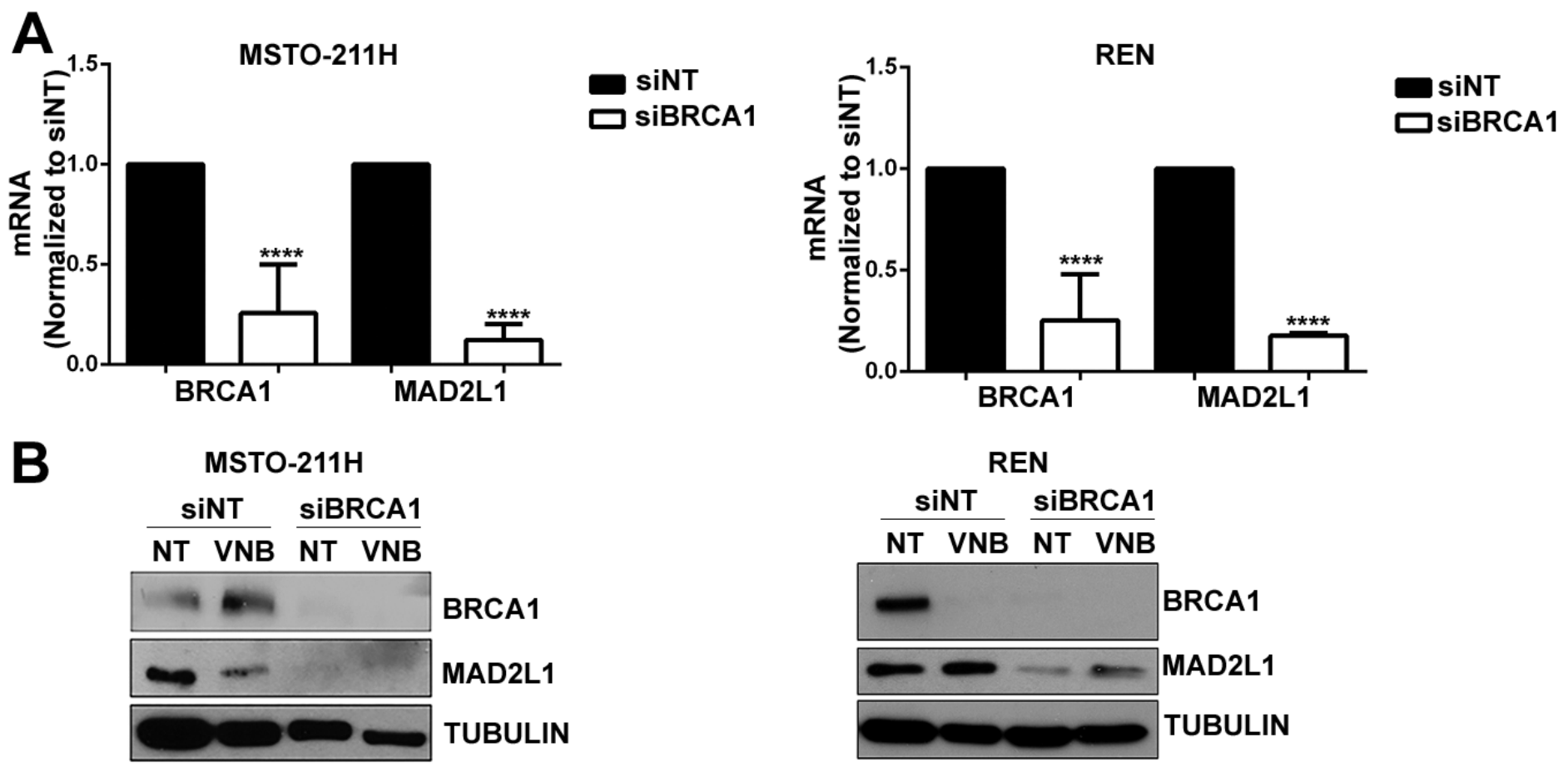

C

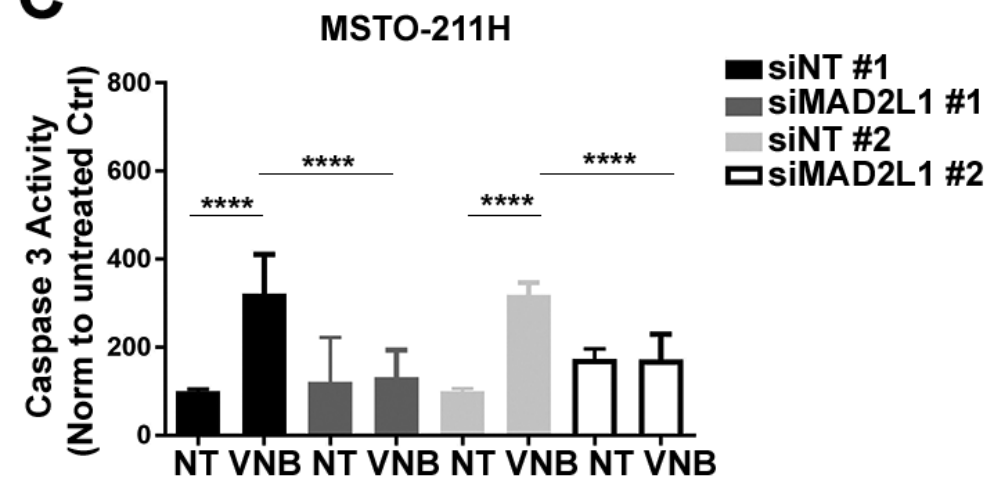

siNT \#1 siMAD2L1 \#1 siNT \#2 siMAD2L1 \#2 NT VNB NT VNB NT VNB NT VNB
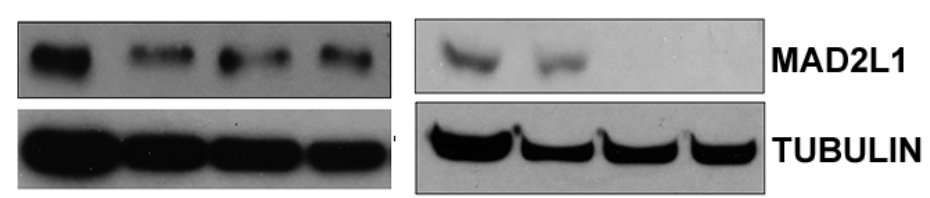

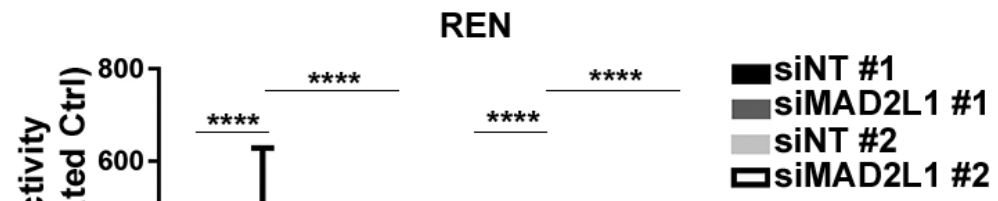

siNT \#1 siMAD2L1 \#1 siNT \#2 siMAD2L1 \#2 NT VNB NT VNB NT VNB NT VNB

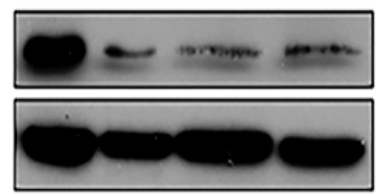

MAD2L1

TUBULIN 
Figure 3
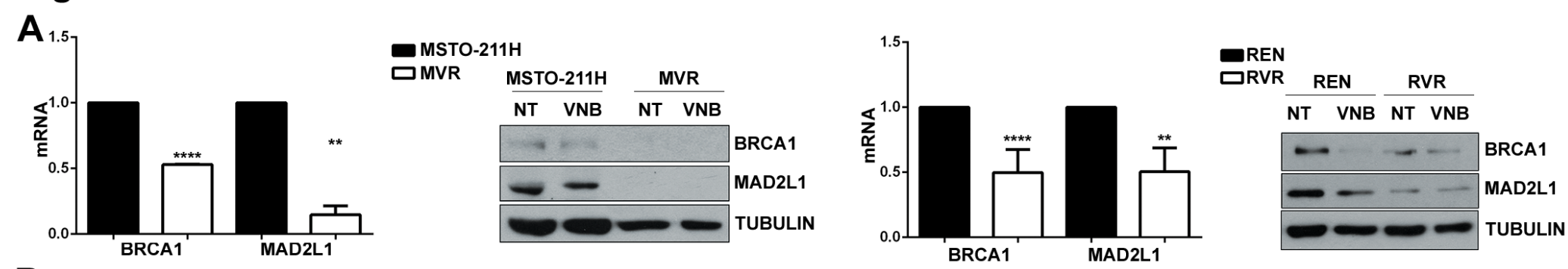

B
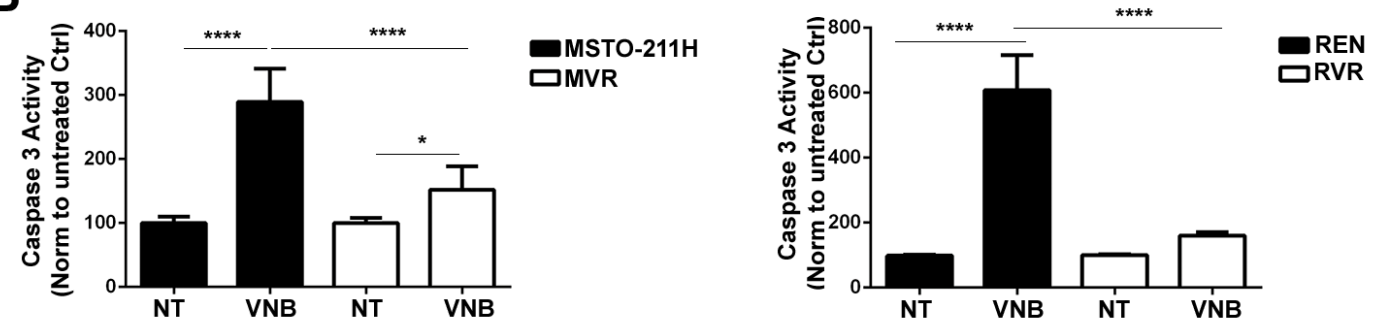

C

Hours
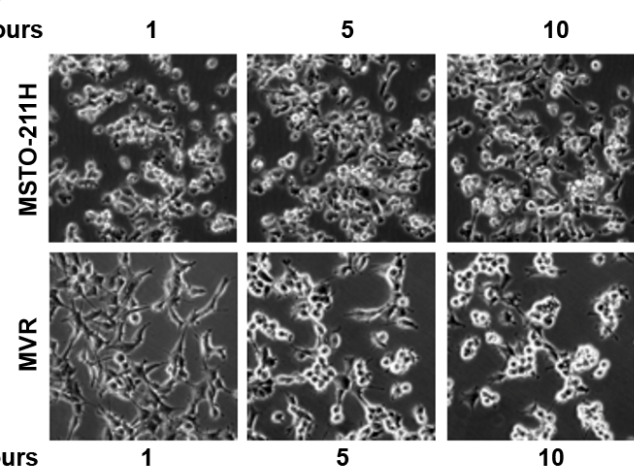

10

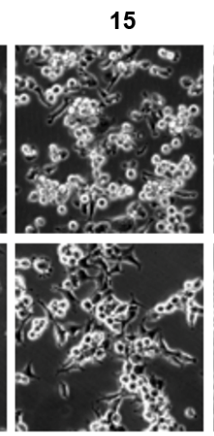

20

25
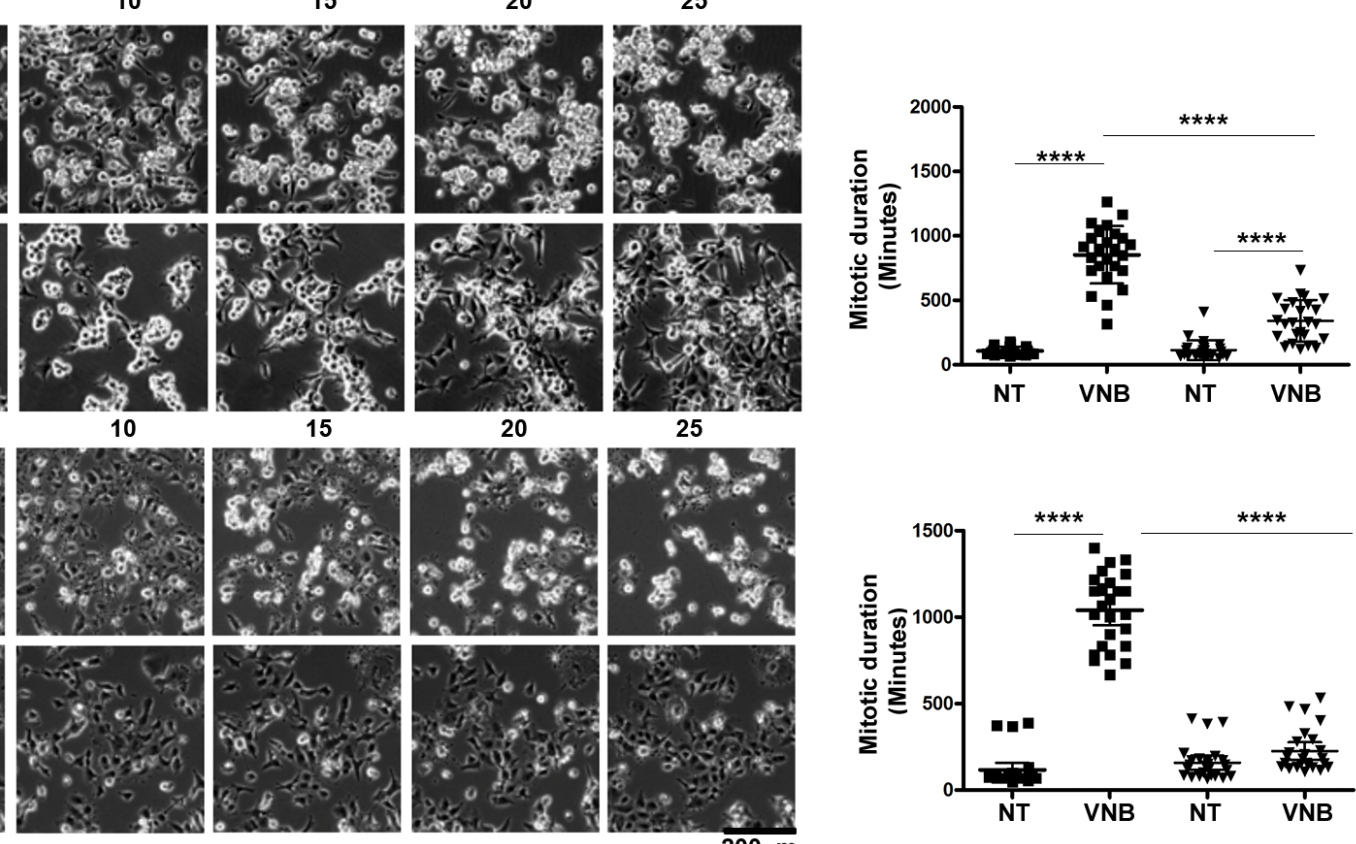

- MSTO-211H

V MVR
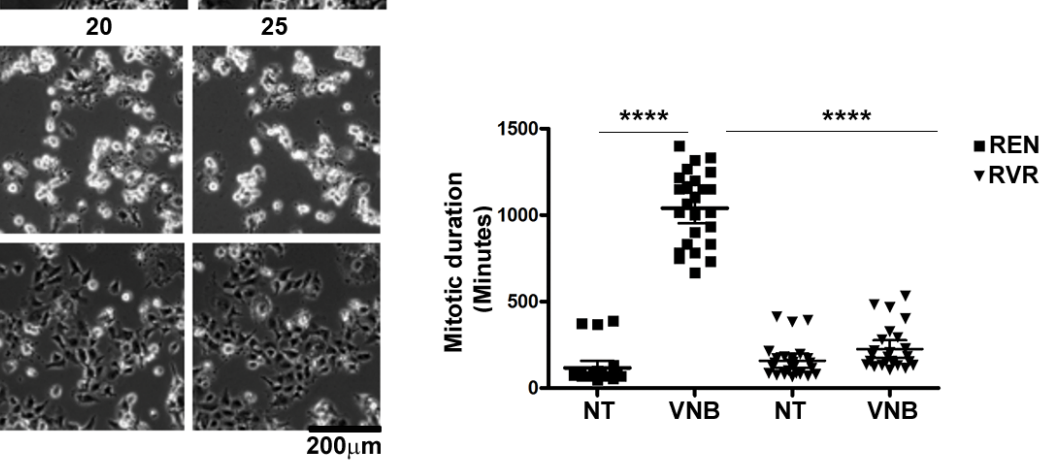

D

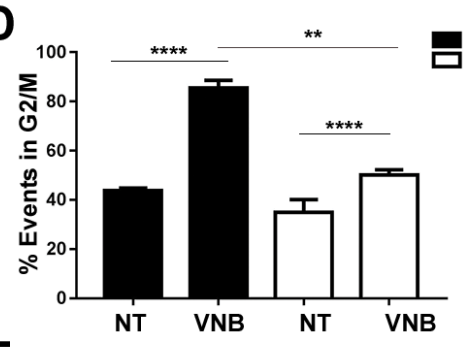

口MSTO-211H

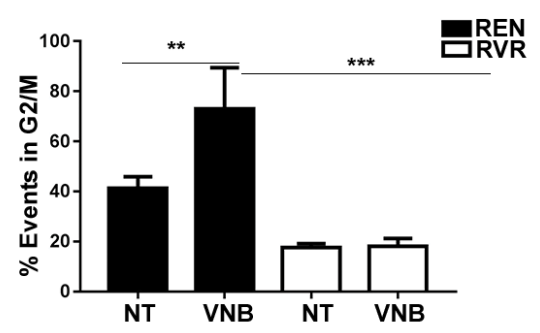

E
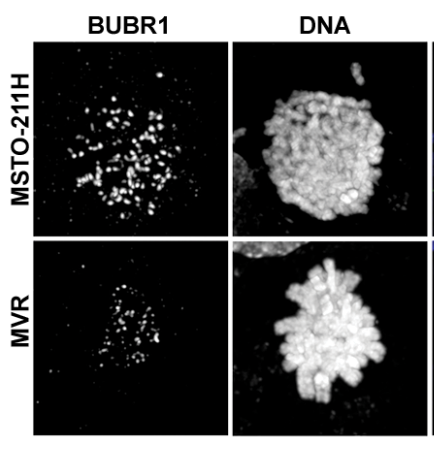

MERGE
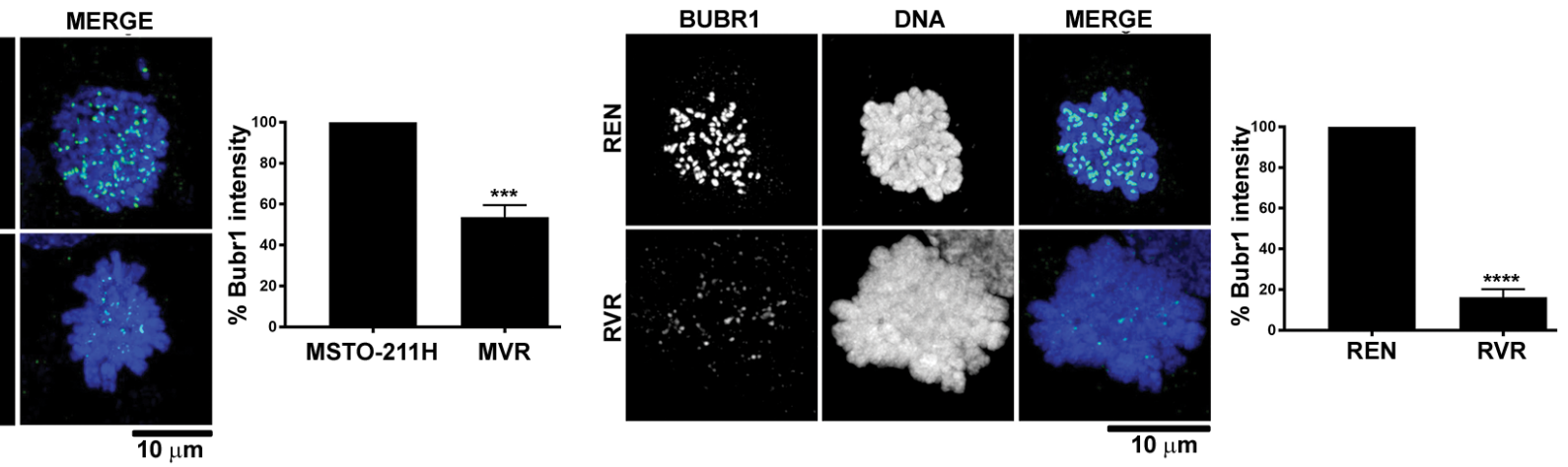

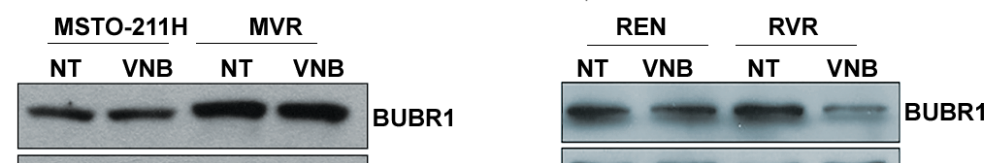


Figure 4

A

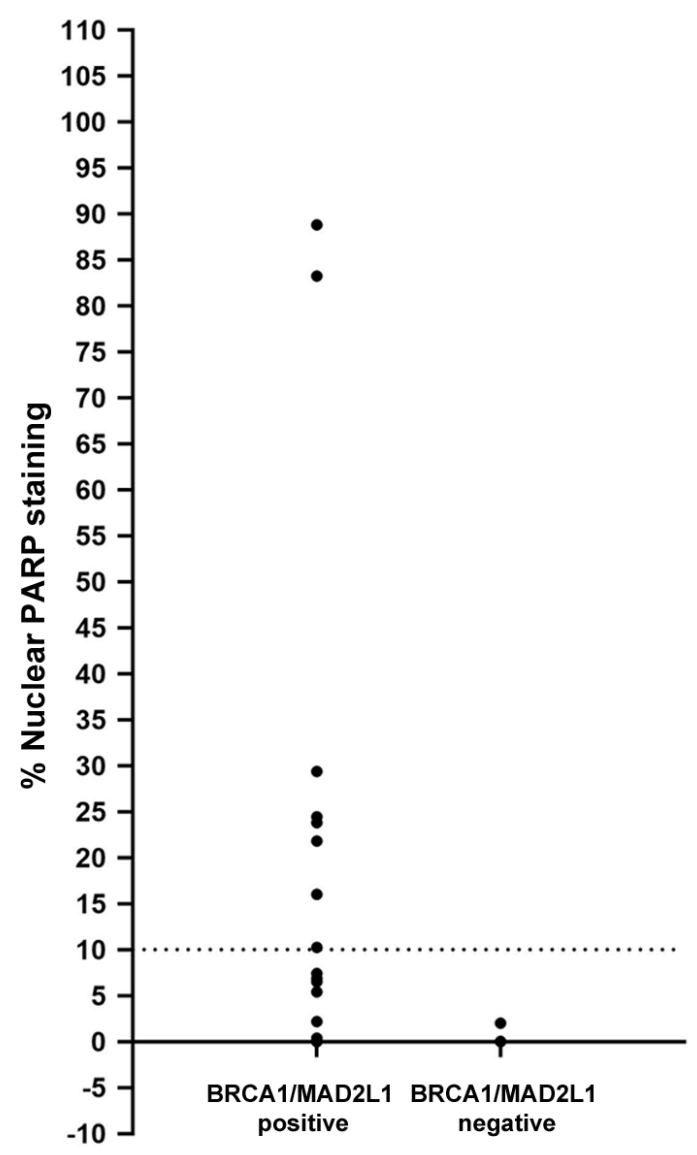

C

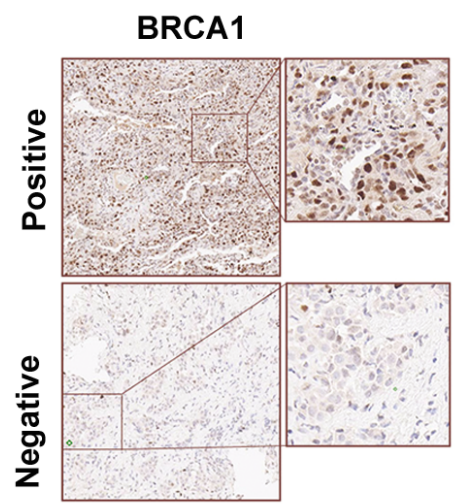

E

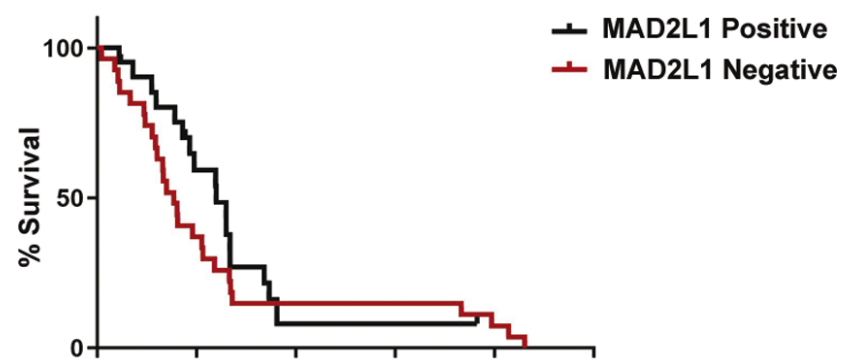

NT

BRCA1

MAD2L1

PARP

BRCA1
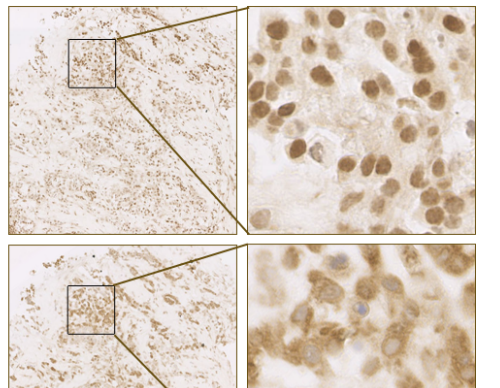

$$
\text { - }
$$

MAD2L1

PARP
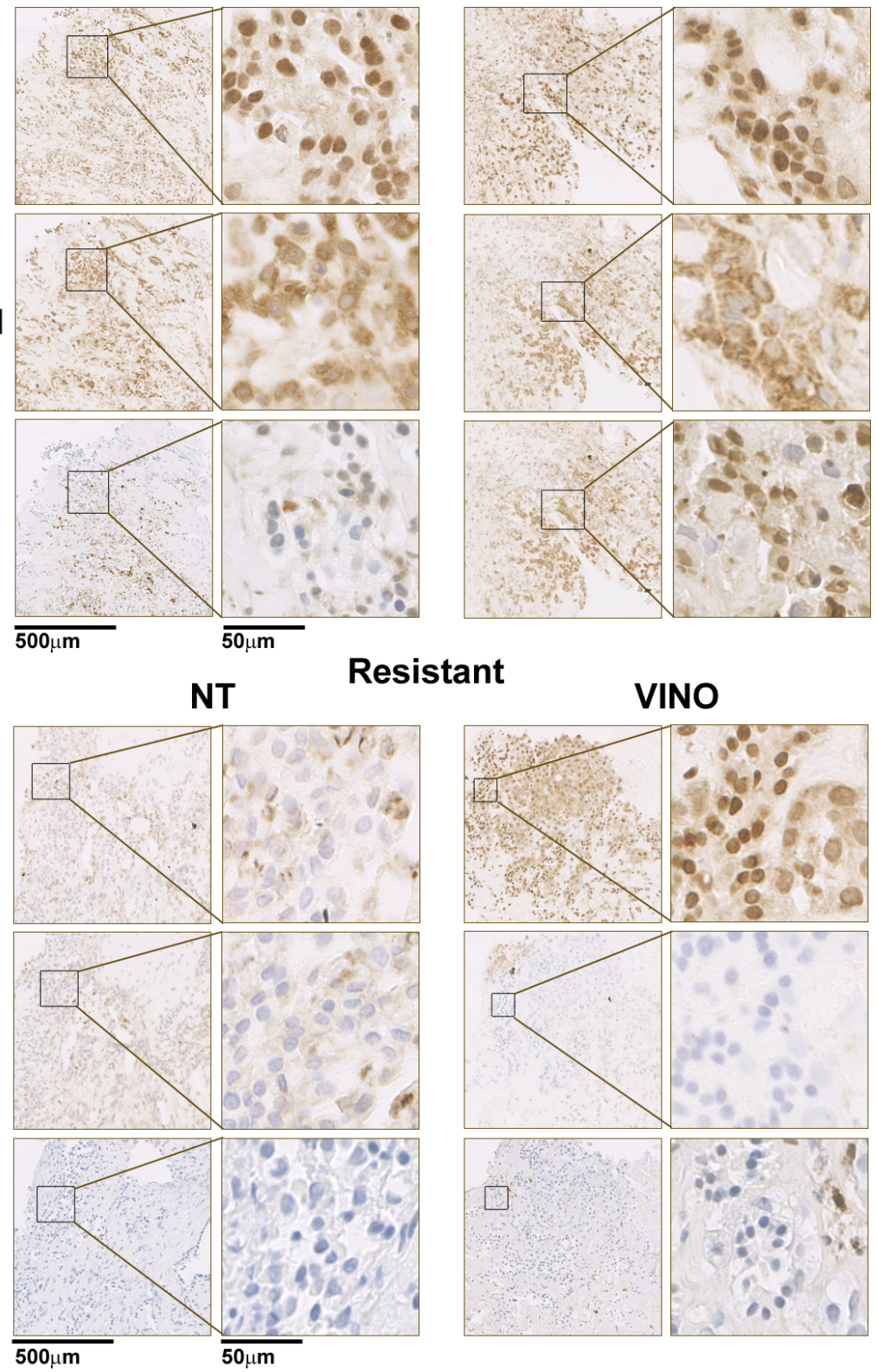

D

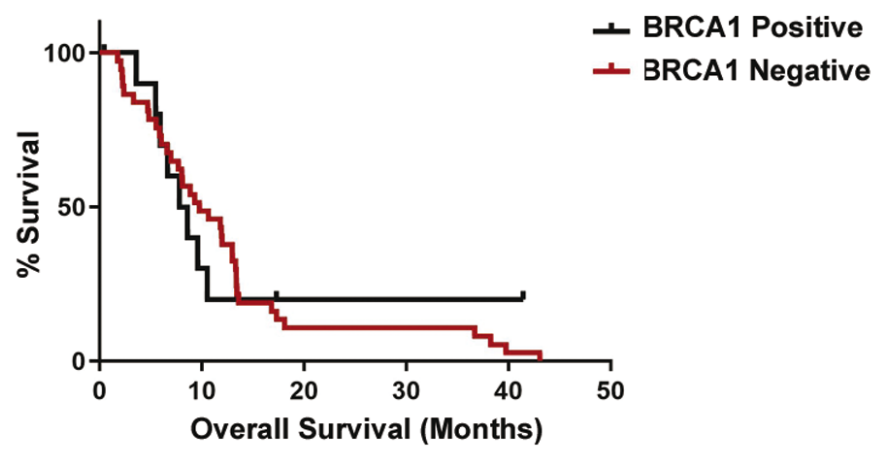

F

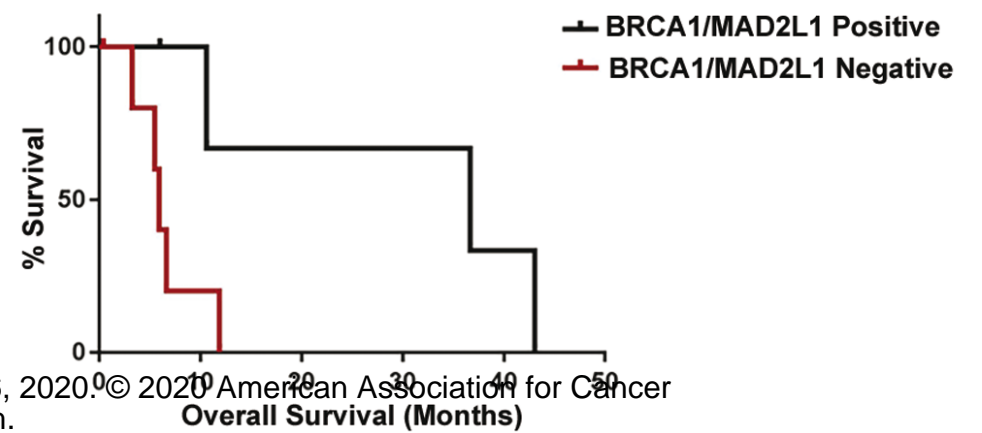




\section{Molecular Cancer Therapeutics}

\section{BRCA1/MAD2L1 deficiency disrupts the spindle assembly checkpoint to confer vinorelbine resistance in mesothelioma}

Sara Busacca, Laura O'Regan, Anita Singh, et al.

Mol Cancer Ther Published OnlineFirst November 6, 2020.

Updated version Access the most recent version of this article at: doi:10.1158/1535-7163.MCT-20-0363

Supplementary Access the most recent supplemental material at:

Material http://mct.aacrjournals.org/content/suppl/2020/11/06/1535-7163.MCT-20-0363.DC1

Author Author manuscripts have been peer reviewed and accepted for publication but have not yet Manuscript been edited.

E-mail alerts Sign up to receive free email-alerts related to this article or journal.

Reprints and Subscriptions

Permissions
To order reprints of this article or to subscribe to the journal, contact the AACR Publications Department at pubs@aacr.org.

To request permission to re-use all or part of this article, use this link http://mct.aacrjournals.org/content/early/2020/11/06/1535-7163.MCT-20-0363.

Click on "Request Permissions" which will take you to the Copyright Clearance Center's (CCC) Rightslink site. 